\title{
Enfermedad carotídea aterosclerosa y enfermedad de sustancia blanca en sujetos sin historia de infarto cerebral o isquemia cerebral transitoria
}

\author{
Erwin Chiquete, ${ }^{1 *}$ Juan José Gómez-Piña, ${ }^{1}$ Guillermo Ramírez-García, ${ }^{1}$ Eduardo Ruiz-Ruiz, ${ }^{1}$ \\ José Domingo Barrientos-Guerra, ${ }^{1}$ Liz Toapanta-Yanchapaxi, ${ }^{1}$ José Alejandro Flórez-Cardona, ${ }^{2}$ \\ Fernando Flores-Silva, ${ }^{1}$ Isael Reyes-Melo, ${ }^{1}$ Guillermo García-Ramos, ${ }^{1}$ Jesús Higuera-Calleja ${ }^{3}$ y \\ Carlos Cantú-Brito ${ }^{1}$ \\ 'Instituto Nacional de Ciencias Médicas y de la Nutrición "Salvador Zubirán", Departamento de Neurología y Psiquiatría, Ciudad de México, México; \\ ${ }^{2}$ Fundación Cardioinfantil-Instituto de Cardiología, Terapia Endovascular Neurológica, Bogotá, Colombia; ${ }^{3}$ Instituto Nacional de Ciencias Médicas \\ y de la Nutrición "Salvador Zubirán", Departamento de Neurorradiología, Ciudad de México, México
}

\section{Resumen}

Introducción: La enfermedad carotídea aterosclerosa (ECA) es un factor de riesgo importante para enfermedad vascular cerebral. Objetivo: Analizar la asociación entre factores de riesgo vascular mayores con ECA y leucopatía cerebral en pacientes sin historia de ictus isquémico. Método: Se evaluaron factores de riesgo en sujetos con exploración de carótidas mediante ultrasonografía Doppler dúplex. No se incluyeron casos con historia de infarto cerebral o ataque isquémico transitorio. Los sujetos contaron con resonancia magnética cerebral y se excluyeron aquellos con lesiones isquémicas de grandes vasos. Se construyeron modelos multivariable para la predicción de ECA, estenosis carotídea significativa, carga de ateromas y leucopatía cerebral. Resultados: Se estudiaron 145 sujetos (60.7\% mujeres, edad de 73 años). Se documentó ECA en $54.5 \%$, estenosis carotídea $\geq 50 \%$ en $9 \%$, carga de placas de ateroma > 6 en $7.6 \%$ y leucopatía periventricular o subcortical en $28.3 \%$ (20.6 \% tenían concurrentemente ECA y leucopatía). Los factores asociados independientemente con ECA fueron edad e hipertensión; con estenosis $\geq 50 \%$, hipertensión; con cargas de ateromas > 6 placas, edad; con leucopatía, edad, diabetes e hipertensión. La obesidad no se asoció con las variables independientes analizadas. Conclusiones: En los sujetos asintomáticos sin historia de ictus isquémico, la edad y la hipertensión fueron los factores de riesgo más importantes para enfermedad macrovascular. La diabetes mellitus se asoció con enfermedad microvascular. La obesidad por sí sola no fue un determinante mayor de ECA o leucopatía cerebral.

PALABRAS CLAVE: Aterosclerosis. Diabetes. Enfermedad carotídea. Hipertensión. Leucopatía. Obesidad.

\section{Abstract}

Introduction: Atherosclerotic carotid artery disease $(C A D)$ is a major risk factor for cerebrovascular disease. Objective: To analyze the association of major vascular risk factors with atherosclerotic CAD and white matter disease (WMD) in patients without a history of ischemic stroke. Method: Risk factors were assessed with carotid examination using Doppler duplex ultrasound. Cases with a history cerebral infarction or transient ischemic attack were not included. Subjects had brain magnetic resonance imaging scans available and those with large-artery ischemic lesions were excluded. Multivariate models were constructed for the prediction of atherosclerotic CAD, significant carotid stenosis, atheroma burden and WMD. Results: One-hundred and forty-five subjects were assessed (60.7\% were females, mean age was 73 years). Atherosclerotic CAD was documented in $54.5 \%$, carotid stenosis $\geq 50 \%$ in $9.0 \%,>6$ atheroma plaques in $7.6 \%$, and periventricular or sub-

Correspondencia:

*Erwin Chiquete

E-mail: erwinchiquete @ hotmail.com
Fecha de recepción: 03-07-2018

Fecha de aceptación: 15-02-2019

DOI: $10.24875 / G M M .19004494$
Gac Med Mex. 2019;155:350-356

Disponible en PubMed

www.gacetamedicademexico.com 
cortical WMD in $28.3 \%$ (20.6\% had atherosclerotic CAD and WMD concurrently). Risk factors independently associated with atherosclerotic CAD were age and hypertension; hypertension was associated with $\geq 50 \%$ carotid stenosis; age was associated with > 6 atheroma plaques; and age, diabetes and hypertension were associated with WMD. Obesity was not associated with any of the analyzed independent variables. Conclusions: In asymptomatic subjects without a history of ischemic stroke, age and hypertension were the most important risk factors for macrovascular disease. Diabetes mellitus was associated with microvascular disease. Obesity alone was not a major determinant of CAD or WMD.

KEY WORDS: Atherosclerosis. Diabetes. Carotid artery disease. Hypertension. White matter disease. Obesity.

\section{Introducción}

La enfermedad carotídea aterosclerosa (ECA) de moderada a severa tiene una prevalencia en la población general asintomática de 0.3 a $6 \%$, dependiendo de la edad y el sexo. ${ }^{1-5}$ Esta frecuencia puede incrementarse notablemente en sujetos con factores de riesgo cardiovascular, pero sobre todo en aquellos con historia de enfermedad aterotrombótica, de tal manera que en grupos de alto riesgo puede documentarse ECA en aproximadamente $80 \%$ de los casos, según el grupo estudiado. ${ }^{6-12}$

La ECA clínicamente significativa puede definirse según el grado de estenosis, el engrosamiento de la pared arterial, la carga de placas de ateroma o sus dimensiones, lo que depende del método usado para documentar la ECA (ultrasonido carotídeo, tomografía, resonancia magnética, autopsia, entre otros). ${ }^{2,4,12} \mathrm{En}$ la población general es más frecuente la ECA clínicamente significativa debida a placas más que a estenosis $>50 \%$; ${ }^{1}$ no obstante su baja frecuencia relativa, la ECA es causa de hasta $30 \%$ de los casos de ictus isquémico (infarto cerebral o ataque isquémico transitorio). 2,6,7,10,11 Más aún, la frecuencia de enfermedad ateromatosa y aterotrombótica en otros lechos arteriales de quienes padecen ECA es muy alta,,$^{13}$ lo mismo que el deterioro de las funciones mentales,${ }^{14}$ de ahí que la importancia clínica de la ECA va más allá de su relación causal con el ictus isquémico.

La relación de factores de riesgo tradicionales y emergentes con ECA en individuos asintomáticos sin historia de ictus ha sido compleja de definir, debido a la metodología para evidenciar ECA, el modelo de estudio epidemiológico y la recolección de las variables. Recientemente se ha documentado que factores de riesgo tales como hipertensión, diabetes mellitus, tabaquismo y dislipidemia son más importantes en su relación con ECA asintomática que la propia obesidad. ${ }^{7,12,15,16}$ Nuestro objetivo fue describir la relación de factores de riesgo tradicionales con ECA en individuos sin historia de ictus.

\section{Método}

Estudio retrospectivo de pacientes referidos para exploración ultrasonográfica de carótidas en el Instituto Nacional de Ciencias Médicas y Nutrición "Salvador Zubirán", Ciudad de México. Fueron seleccionados los pacientes que cumplieran con los siguientes criterios:

- Adultos de uno u otro sexo.

- Evaluación ultrasonográfica de carótidas por razón diferente a la búsqueda de etiología de enfermedad vascular cerebral.

- Sin historia personal de ictus hemorrágico 0 isquémico (infarto cerebral 0 ataque isquémico transitorio).

- Contar con estudio de resonancia magnética de encéfalo seis meses antes o después de la realización del ultrasonograma de las carótidas.

Se excluyeron los casos en los que se documentó cualquiera de los criterios siguientes:

- Examen físico con signos de focalización neurológica.

- Demencia.

- Estudios de neuroimagen con lesiones corticosubcorticales típicas de infarto cerebral de grandes vasos.

Los comités de investigación y de ética en investigación del centro aprobaron este estudio. Se empleó un instrumento físico de captura estandarizado para recabar los datos que integraron una base de datos electrónica. Del expediente clínico se documentaron características demográficas, factores de riesgo cardiovascular, tratamientos médicos y características de la ultrasonografía dúplex de arterias cervicales.

Se definió como estenosis significativa, la presencia de placa de ateroma en la carótida interna que provoca estenosis $\geq 50 \%$.,6,7 De cada paciente se revisó la ultrasonografía carotídea para caracterizar la carga de placas de ateroma y el grado de estenosis identificable por este método. La carga de placas fue categorizada de acuerdo con su número: leve, una a 
tres placas; moderada, cuatro a seis placas y severa, $\geq 7$ placas.

Se definió como ECA a la presencia de cualquiera de las siguientes características: una o más placas de ateroma, grosor de íntima media carotídea $\geq 1 \mathrm{~mm}$ o estenosis carotídea $\geq 50 \%$.

Además, se recolectaron variables de la imagen por resonancia magnética (IRM) de encéfalo, tales como enfermedad de sustancia blanca, su distribución y extensión. Fueron excluidos los casos en que se identificaron potenciales lesiones isquémicas. Se definió enfermedad de sustancia blanca a la presencia de aumento en la intensidad de señal en secuencias T2 y FLAIR en regiones periventricular o subcortical.

Las frecuencias relativas simples de variables nominales se expresan en forma de proporciones y la edad en mediana con rango intercuartílico, ya que no presentó distribución normal. Para comparar las frecuencias de variables nominales entre dos o más grupos se usó chi cuadrada de Pearson. Las medianas de variables cuantitativas no paramétricas fueron comparadas entre dos grupos mediante $U$ de Mann-Whitney. Se construyeron modelos multivariables mediante regresión logística binaria para identificar factores de riesgo potencialmente asociados con estenosis carotídea significativa, carga de placas de ateroma y enfermedad de sustancia blanca en la IRM, para lo cual se seleccionaron las variables que se asociaran con la variable dependiente en el análisis bivariable, con $p<0.20$. Los resultados del análisis multivariable se registraron con razón de momios (RM) y sus intervalos de confianza (IC) de $95 \%$. Para evaluar la confiabilidad de los modelos se empleó la prueba de bondad de ajuste de Hosmer y Lemeshow; se consideró adecuada cuando se obtuvo $p>0.20$. Todos los valores de $p$ para comparaciones fueron calculados a dos colas y considerados significativos cuando fueron < 0.05. El paquete estadístico SPSS versión 24.0 fue usado en todos los cálculos.

\section{Resultados}

De 194 pacientes seleccionados inicialmente, fueron excluidos 49 después de aplicar los criterios de selección. Así, 145 pacientes fueron incluidos en el análisis final $(60.7 \%$ mujeres, mediana de la edad: 73 años, rango intercuartílico = 63-81 años. Las razones por las que fue practicada la ultrasnografía dúplex de carótidas y neuroimagen fueron la evaluación de síncope (63\%), deterioro cognoscitivo leve o deterioro de memoria (22\%), enfermedad reumatológica (8\%), cardiopatía isquémica ( $2 \%$ y otros motivos ( $5 \%$ ). Ningún paciente de la base final contaba con antecedente de ictus isquémico, examen físico con focalización neurológica o neuroimagen con lesiones isquémicas de grandes vasos.

Los factores de riesgo más frecuentes fueron edad $\geq 65$ años ( $n=103,71 \%$ ), hipertensión ( $n=91$, $62.8 \%$ ), hipercolesterolemia ( $n=66,45.5 \%$ ), tabaquismo ( $n=59,40.7 \%$ ), diabetes mellitus ( $n=52$, $35.9 \%$ ) y obesidad ( $n=48,33.1 \% \%$ ). La distribución de factores de riesgo fue comparable entre hombres y mujeres, excepto para tabaquismo (56.1 y $30.7 \%$, respectivamente; $p=0.002)$, hipertensión arterial (52.6 y $69.3 \%, p=0.042)$ y diabetes mellitus (29.5 y $45.6 \%, p=0.049)$.

La prevalencia de ECA fue de $54.5 \%(n=79)$, de estenosis carotídea $\geq 50 \%$ de $9.0 \%(n=13)$ y de leucopatía periventricular o subcortical en la IRM de $28.3 \%(n=41) ; 30$ pacientes $(20.7 \%)$ presentaron concurrencia de ECA y leucopatía, es decir, $73.1 \%$ del total que presentaba leucopatía. La frecuencia de ECA entre hombres y mujeres no fue distinta (55.7 y $52.6 \%, p=0.719)$, tampoco la de estenosis carotídea $\geq 50 \%$ (7.0 y $10.2 \%, p=0.509)$ o la de leucopatía (19.3 y $34.1 \%, p=0.053)$. No obstante, la prevalencia de la coexistencia de ECA con leucopatía sí fue significativamente mayor en las mujeres $(26.1 \%)$ que en los hombres (12.3\%) ( $p=0.044)$.

En el análisis bivariable se identificaron como factores de riesgo asociados a ECA a la edad, hipertensión y diabetes, y potencialmente como asociados la hipercolesterolemia, cardiopatía isquémica y fibrilación auricular (Tabla 1). Los factores asociados con leucopatía fueron la edad, hipertensión y diabetes, y potencialmente el sexo femenino. Los factores de riesgo estadísticamente significativos y los potenciales $(p<0.20)$ integraron modelos multivariables para la predicción de ECA, estenosis carotídea $\geq 50 \%$ y carga de placas de ateroma $>6$ (Tabla 2). Fue así como se identificó a la edad y a la hipertensión como factores independientes de riesgo para ECA, a la hipertensión como único factor independiente asociado con estenosis carotídea $\geq 50 \%$ y a la edad como único factor independiente de predicción de carga ateromatosa alta. En el modelo de predicción de enfermedad de sustancia blanca se identificó a la edad, diabetes e hipertensión como las variables independientes en la predicción del riesgo (Tabla 3 ). Estas mismas variables se asociaron de forma independiente con la concurrencia de leucopatía con ECA (Tabla 4). 
Tabla 1. Análisis bivariable sobre factores de riesgo vascular mayores asociados con enfermedades carotídea y de pequeño vaso*

\begin{tabular}{|c|c|c|c|c|c|c|c|c|c|c|}
\hline \multirow[t]{3}{*}{ Factores de riesgo } & \multicolumn{4}{|c|}{ Enfermedad carotídea } & \multirow[t]{3}{*}{ p } & \multicolumn{4}{|c|}{ Enfermedad de pequeño vaso } & \multirow[t]{3}{*}{$p$} \\
\hline & \multicolumn{2}{|c|}{$\underset{(n=66)}{\operatorname{Sin}}$} & \multicolumn{2}{|c|}{$\begin{array}{c}\text { Con } \\
(n=79)\end{array}$} & & \multicolumn{2}{|c|}{$\underset{\substack{\operatorname{Sin} \\
(n=104)}}{ }$} & \multicolumn{2}{|c|}{$\begin{array}{c}\text { Con } \\
(n=41)\end{array}$} & \\
\hline & Mediana & RIC & Mediana & $\%$ & & Mediana & $\%$ & Mediana & RIC & \\
\hline \multirow[t]{2}{*}{ Edad en años } & 67 & $56-77$ & 76 & $69-83$ & $<0.001$ & 69 & $60-77$ & 81 & $74-84$ & $<0.001$ \\
\hline & $\mathrm{n}$ & $\%$ & $\mathrm{n}$ & $\%$ & & $\mathrm{n}$ & $\%$ & $\mathrm{n}$ & $\%$ & \\
\hline Sexo femenino & 39 & 59.1 & 49 & 62.0 & 0.719 & 58 & 55.8 & 30 & 73.2 & 0.053 \\
\hline $\mathrm{IMC} \geq 27$ & 21 & 31.8 & 27 & 34.2 & 0.764 & 36 & 34.6 & 12 & 29.3 & 0.538 \\
\hline $\mathrm{IMC} \geq 30$ & 12 & 18.2 & 12 & 15.2 & 0.629 & 18 & 17.3 & 6 & 80.5 & 0.696 \\
\hline Hipertensión & 31 & 47.0 & 60 & 75.9 & $<0.001$ & 58 & 55.8 & 33 & 80.5 & 0.006 \\
\hline Hipercolesterolemia & 26 & 39.4 & 40 & 50.6 & 0.176 & 45 & 43.3 & 21 & 51.2 & 0.387 \\
\hline Hipertrigliceridemia & 18 & 27.3 & 23 & 29.1 & 0.806 & 28 & 26.9 & 13 & 31.7 & 0.565 \\
\hline Dislipidemia mixta** & 17 & 25.8 & 19 & 24.1 & 0.813 & 25 & 24.0 & 11 & 26.8 & 0.726 \\
\hline Diabetes & 16 & 24.2 & 36 & 45.6 & 0.008 & 30 & 28.8 & 22 & 53.7 & 0.005 \\
\hline Tabaquismo*** & 27 & 40.9 & 32 & 40.5 & 0.961 & 42 & 40.4 & 17 & 41.5 & 0.905 \\
\hline Cardiopatía isquémica & 4 & 6.1 & 10 & 12.7 & 0.180 & 9 & 8.7 & 5 & 12.2 & 0.516 \\
\hline Fibrilación auricular no valvular & 1 & 1.5 & 6 & 7.6 & 0.089 & 4 & 3.8 & 3 & 7.3 & 0.380 \\
\hline
\end{tabular}

*Enfermedad carotídea aterosclerosa definida aquí como cualquiera de las siguientes: una o más placas de ateroma, grosor íntima-media carotídea $\geq 1$ mm o estenosis carotídea $\geq 50 \%$. Enfermedad de sustancia blanca en resonancia magnética caracterizada por aumento en la intensidad de señal en secuencias T2 y FLAIR en regiones periventricular o subcortical.

${ }^{* *}$ Hipercolesterolemia e hipertrigliceridemia.

${ }^{\star \star \star}$ Tabaquismo actual o previo. RIC = rango intercuartilar.

Tabla 2. Enfermedad de grandes vasos: modelos multivariable sobre factores de riesgo vascular asociados con estenosis carotídea, carga de ateroma y enfermedad de sustancia blanca $(n=194)$

\begin{tabular}{|l|l|l|}
\hline Modelo & Variable & RM (IC 95\%) \\
\hline Modelo para enfermedad carotídea aterosclerosa* & Edad** & $1.070(1.035-1.106)$ \\
\hline Modelo para estenosis carotídea moderada a severa** & Hipertensión & H.628 (1.681-7.830) \\
\hline Modelo para carga de placas de ateroma $>6^{\ddagger}$ & Hipertensión & $8.051(1.016-63.766)$ \\
\hline
\end{tabular}

*Enfermedad carotídea aterosclerosa definida como cualquiera de las siguientes: una o más placas de ateroma, grosor íntima-media carotídeo $\geq 1$ mm o estenosis carotídea $\geq 50$ \%. Prueba de Hosmer-Lemeshow para bondad de ajuste en el último paso: $\chi^{2}=5.094,2 \mathrm{gl}, \mathrm{p}=0.748$. Modelo ajustado por edad, sexo, diabetes, hipertensión, obesidad, tabaquismo, hipercolesterolemia, hipertrigliceridemia, fibrilación auricular, historia personal de cardiopatía isquémica e historia familiar de eventos aterotrombóticos.

${ }^{\star *}$ Edad por cada año de incremento.

${ }^{* * *}$ Estenosis carotídea $\geq 50 \%$. Prueba de Hosmer-Lemeshow para bondad de ajuste en el último paso: $\chi^{2}=3,473,1 \mathrm{gl}, \mathrm{p}=0.812$. Modelo ajustado por edad, sexo, diabetes hipertensión, obesidad, tabaquismo, hipercolesterolemia, hipertrigliceridemia, fibrilación auricular, historia personal de cardiopatía isquémica e historia familiar de eventos aterotrombóticos.

łPrueba de Hosmer-Lemeshow para bondad de ajuste en el último paso: $\chi^{2}=3,468,1 \mathrm{gl}, \mathrm{p}=0.839$. Modelo ajustado por edad, sexo, diabetes, hipertensión, obesidad, tabaquismo, hipercolesterolemia, hipertrigliceridemia, fibrilación auricular, historia personal de cardiopatía isquémica e historia familiar de eventos aterotrombóticos.

Tabla 3. Enfermedad de pequeño vaso: modelo multivariable sobre factores de riesgo asociados con enfermedad de sustancia blanca en individuos sin historia de ictus isquémico $(n=194)$

\begin{tabular}{|l|l|l|l|}
\hline Modelo & Variable & RM (IC 95 \%) & \multicolumn{1}{c|}{$\mathbf{p}$} \\
\hline Modelo para enfermedad de sustancia blanca en resonancia magnética* & Edad $^{* *}$ & $1.121(1.066-1.179)$ & 0.002 \\
\hline & Diabetes mellitus & $2.425(1.035-5.681)$ & 0.005 \\
\hline & Hipertensión & $3.896(1.416-10.719)$ & 0.005 \\
\hline
\end{tabular}

*Enfermedad de sustancia blanca en resonancia magnética caracterizada por aumento en la intensidad de señal en secuencias T2 y FLAIR en regiones periventricular o subcortical. Prueba de Hosmer-Lemeshow para bondad de ajuste en el último paso: $\chi^{2}=2.752,3 \mathrm{gl}, \mathrm{p}=0.907$. Modelo ajustado por edad, sexo, diabetes, hipertensión, obesidad, tabaquismo, hipercolesterolemia, hipertrigliceridemia, fibrilación auricular, historia personal de cardiopatía isquémica e historia familiar de eventos aterotrombóticos.

**Edad por cada año de incremento. 
Tabla 4. Coexistencia de enfermedades de grandes y pequeños vasos: modelo multivariable sobre factores de riesgo asociados con concurrencia de enfermedad ateromatosa carotídea y enfermedad de sustancia blanca en individuos sin historia de ictus isquémico $(n=194)$

\begin{tabular}{|c|c|c|c|}
\hline Modelo & Variable & RM (IC $95 \%)$ & $p$ \\
\hline \multirow{3}{*}{$\begin{array}{l}\text { Modelo para la concurrencia de enfermedad ateromatosa carotídea y enfermedad de } \\
\text { sustancia blanca* }\end{array}$} & Edad $^{\star *}$ & $1.114(1.052-1.179)$ & $<0.001$ \\
\hline & Diabetes mellitus & $3.967(1.533-10.268)$ & 0.005 \\
\hline & Hipertensión & $6.090(1.728-21.468)$ & 0.005 \\
\hline
\end{tabular}

*Enfermedad carotídea aterosclerosa definida como cualquiera de las siguientes: una o más placas de ateroma, grosor de íntima-media carotídea $\geq 1 \mathrm{~mm}$ o estenosis carotídea $\geq 50$ \%. Enfermedad de sustancia blanca en resonancia magnética caracterizada por aumento en la intensidad de señal en secuencias T2 y FLAIR en regiones periventricular o subcortical. Prueba de Hosmer-Lemeshow para bondad de ajuste en el último paso: $\chi^{2}=2.890,3 \mathrm{gl}, \mathrm{p}=0.941$. Modelo ajustado por edad, sexo, diabetes, hipertensión, obesidad, tabaquismo, hipercolesterolemia, hipertrigliceridemia, fibrilación auricular, historia personal de cardiopatía isquémica e historia familiar de eventos aterotrombóticos.

**Edad por cada año de incremento.

\section{Discusión}

La ECA se divide clínicamente en estenosante y no estenosante según el grado de reducción de la luz del vaso. ${ }^{17,18}$ Aunque la ECA estenosante es la patología carotídea que más se relaciona causalmente con el ictus isquémico, es fundamental considerar que la aterosclerosis indica arteria no sana y que se relaciona con mucha frecuencia a enfermedad ateromatosa en otros lechos arteriales,,$^{12,15,19}$ lo que puede dar lugar a confusiones en la literatura médica y en la práctica clínica rutinaria, al considerar erróneamente una arteria normal cuando no presenta estenosis. ${ }^{20,21}$ La ECA, aún en su forma no estenosante, es un conocido marcador de riesgo tanto de ictus isquémico como de muerte por eventos aterotrombóticos. ${ }^{19,22-26}$ Por ello, es importante identificar la relación entre los factores de riesgo vascular y las distintas dimensiones o características de la ECA, especialmente en individuos sin historia de ictus, con el fin de evitar conclusiones erróneas derivadas del fenómeno de causalidad reversa cuando se estudia solo a pacientes con ictus secundario a enfermedad de grandes vasos.

Como ha demostrado el presente estudio, la ECA se asocia a su vez de formas diferentes con factores de riesgo tradicionales llamados "mayores", dependiendo del criterio o características de la enfermedad ateromatosa. ${ }^{1,9,14,25-29}$ Un caso similar puede describirse en la enfermedad de la sustancia blanca cerebral (por ejempo, leucopatía o leucoaraiosis), según se defina mediante tomografía o IRM y de acuerdo con características de su distribución y extensión. ${ }^{29-31}$ Como se ha informado, ${ }^{1,14}$ en el presente análisis no se encontró que la obesidad fuese un factor de riesgo independiente de ECA o leucopatía. Es posible que la obesidad se relacione causalmente más con dislipidemia, diabetes 0 hipertensión que de forma directa con la enfermedad macro y microvascular. También es posible que este estudio no haya demostrado una asociación directa e independiente entre la obesidad y la arteriopatía por un poder estadístico limitado, o porque el índice de masa corporal (IMC) no sea un marcador antropométrico ideal de adiposidad, como actualmente se ha reconocido. ${ }^{32-34}$ En nuestra serie, el factor de riesgo más importante fue la hipertensión, lo que es congruente con lo informado con anterioridad, ${ }^{25-31}$ mientras que la diabetes fue un factor de riesgo más asociado con microangiopatía cerebral, al menos en el grupo estudiado.

Interesantemente, aunque con anterioridad se consideraba que la ECA y la enfermedad de pequeño vaso no se encontraban vinculadas, ${ }^{35}$ ahora se sabe que no solo comparten factores de riesgo, sino que con frecuencia coexisten ambas entidades. ${ }^{32,35} \mathrm{Es}$ importante hacer notar que la distribución de los factores de riesgo en poblaciones de bajo riesgo (por ejemplo, población general o asintomáticos) tiende a diferir entre los distintos grupos étnicos, mientras que es relativamente homogénea cuando se estudia a poblaciones de alto riesgo (por ejemplo, con eventos aterotrombóticos). ${ }^{1,10,14,36}$ Así, es posible que los factores de riesgo identificados difieran de los de otros grupos de individuos asintomáticos.

Este estudio presenta potenciales limitaciones que deben señalarse, la más significativa es el tamaño de muestra relativamente bajo para la detección de factores de riesgo, cuya frecuencia es baja, pero cuya asociación causal pudiese ser alta para el desarrollo de macro y microangiopatía. Por otro lado, la naturaleza retrospectiva del estudio podría implicar sesgos de selección, considerando las indicaciones médicas que motivaron la realización de los estudios de ultrasonografía y neuroimagen.

Al tratarse de un estudio transversal, no ha sido posible evaluar la implicación de los marcadores de enfermedad macro y microvascular con desenlaces 
clínicos de importancia, tales como ictus isquémico, deterioro cognoscitivo mayor y muerte cardiovascular. Más aún, si bien se cuidó seleccionar sujetos asintomáticos, al ser pacientes y participantes no voluntarios de una institución de referencia no pueden ser considerados representantes de la población general asintomática.

No obstante lo anterior, este análisis aporta información útil en la generación de nuevas hipótesis científicas, provee datos fundamentales para el cálculo de la muestra de próximos estudios y apoya en la toma de decisiones clínicas, particularmente respecto a la búsqueda de enfermedad arterial y metabólica entre quienes presentan ECA o leucopatía vascular.

En conclusión, en este grupo de sujetos asintomáticos sin historia de enfermedad cerebrovascular aguda, la edad y la hipertensión fueron los factores de riesgo más importantes relacionados con imagen de enfermedad macrovascular. La diabetes mellitus se asoció más con enfermedad microvascular y la obesidad per se no fue un determinante mayor de ECA o de microangiopatía cerebral.

\section{Declaraciones de potenciales conflictos de intereses}

No existió afiliación alguna con organizaciones con un interés monetario o ético directo o indirecto con la sustancia de lo tratado en este escrito científico, por lo que todos los autores declaran no tener conflictos de interés que afectaran el diseño y reporte de los resultados del estudio.

\section{Bibliografía}

1. Woo SY, Joh JH, Han SA, Park HC. Prevalence and risk factors for atherosclerotic carotid stenosis and plaque: a population-based screening study. Medicine (Baltimore). 2017:96:e5999.

2. Gómez-Díaz RA, Gutiérrez J, Contreras-Rodríguez A, Valladares-Salgado A, Tanus-Hajj J, Mondragón-González R, et al. Association of V249 and T280M variants of fractalkine receptor CX3CR1 with carotid intima-media thickness in a Mexican population with type 2 diabetes. Gac Med Mex. 2017;153:49-56.

3. De Weerd M, Greving JP, Hedblad B, Lorenz MW, Mathiesen EB, O'Leary $\mathrm{DH}$, et al. Prevalence of asymptomatic carotid artery stenosis in the general population: an individual participant data meta-analysis. Stroke. 2010;41:1294-1297.

4. Santos-Franco JA, Zenteno-Castellanos MA, Jaramillo-Magaña JJ, Vega-Montesinos S, Lee-Aguirre A. Endovascular management of carotid atherosclerosis. Section II: current therapeutic approaches and limitations. Gac Med Mex. 2009:145:415-425.

5. Brown HA, Lawrence-Wright MB, Shah S, Lawrence SG, Gilbert D, Crandon I. Prevalence of carotid stenosis in a high-risk Caribbean population. Stroke. 2009:40:1892-1893.

6. Cantú-Brito C, Ruiz-Sandoval Jl, Arauz-Góngora A, Villarreal-Careaga J, Barinagarrementería F, Murillo-Bonilla LM, et al. Prevalencia de estenosis carotídea en pacientes con isquemia cerebral transitoria en México. Rev Mex Neuroci. 2010;11:343-348.

7. Chiquete E, Torres-Octavo B, Cano-Nigenda V, Valle-Rojas D, Domínguez-Moreno $\mathrm{R}$, Tolosa-Tort $\mathrm{P}$, et al. Characterisation of factors associated with carotid stenosis in a population at high risk. Rev Neurol. 2014:58:541-547.

8. Park JH, Kim WH, Kim JH, Park TS, Baek HS. Prevalence of and risk factors for extracranial internal carotid artery stenosis in Korean type 2 diabetic patients. Diabet Med. 2006;23:1377-1380.

9. Araki Y, Kumakura H, Kanai H, Kasama S, Sumino H, Ichikawa A, et al. Prevalence and risk factors for cerebral infarction and carotid artery stenosis in peripheral arterial disease. Atherosclerosis. 2012;223:473-437.

10. Mathiesen EB, Joakimsen O, Bønaa KH. Prevalence of and risk factors associated with carotid artery stenosis: the Tromsø Study. Cerebrovasc Dis. 2001;12:44-51.

11. Wanamaker KM, Moraca RJ, Nitzberg D, Magovern GJ. Contemporary incidence and risk factors for carotid artery disease in patients referred for coronary artery bypass surgery. J Cardiothorac Surg. 2012;7:78.

12. Rodríguez-Flores M, Rodríguez-Saldaña J, Cantú-Brito C, Aguirre-García J, Alejandro GG. Prevalence and severity of atherosclerosis in different arterial territories and its relation with obesity. Cardiovasc Pathol. 2013;22:332-338

13. Zenteno-Castellanos MA, Santos-Franco JA, Vega-Montesinos S, Lee-Aguirre A. Endovascular management of carotid atherosclerosis. Section I: basic considerations and diagnostic elements. Gac Med Mex. 2009:145:407-414

14. Imahori $Y$, Mathiesen EB, Leon DA, Hopstock LA, Hughes AD, Johnsen $\mathrm{SH}$, et al. The contribution of obesity to carotid atherosclerotic plaque burden in a general population sample in Norway: The Tromsø Study. Atherosclerosis. 2018;273:15-20.

15. Chiquete E, Valle-Rojas D, Rodríguez-Saldaña J, Rodríguez-Flores M, Aguirre-García J, Flores-Silva F, et al. Aterosclerosis carotídea e intracraneal en una población pediátrica: un estudio de autopsia. Rev Mex Neuroci. 2012;13:93-97

16. Escobedo J, Schargrodsky H, Champagne B, Silva H, Boissonnet CP, Vinueza R, et al. Prevalence of the metabolic syndrome in Latin Ameri$\mathrm{ca}$ and its association with sub-clinical carotid atherosclerosis: the CARMELA cross sectional study. Cardiovasc Diabetol. 2009;8:52.

17. Eliasziw M, Rankin RN, Fox AJ. Accuracy and prognostic consequences of ultrasonography in identifying severe carotid artery stenosis. North American Symptomatic Carotid Endarterectomy Trial (NASCET) Group. Stroke. 1995;26:1747-1752.

18. Cantú-Brito C. Atherosclerotic carotid stenosis. How to decide endarterectomy versus stenting versus only medical treatment? Rev Invest Clin. 2009;61:53-65.

19. Cantú-Brito C, Chiquete E, Ruiz-Sandoval JL, Gaxiola E, Albuquerque DC Corbalán $\mathrm{R}$, et al. Atherothrombotic disease, traditional risk factors, and 4-year mortality in a Latin American population: the REACH Registry. Clin Cardiol. 2012;35:451-457.

20. Joakimsen O, Bonaa KH, Mathiesen EB, Stensland-Bugge E, Arnesen E. Prediction of mortality by ultrasound screening of a general population for carotid stenosis: the Tromsø Study. Stroke. 2000;31:1871-1876.

21. Kim AH, Augustin G, Shevitz A, Kim H, Trivonovich MR, Powell AR, et al. Carotid consensus panel duplex criteria can replace modified University of Washington criteria without affecting accuracy. Vasc Med. 2018;23: 126-133.

22. Waltimo $\mathrm{O}$, Kaste $\mathrm{M}$, Fogelholm $\mathrm{R}$. Prognosis of patients with unilateral extracranial occlusion of the internal carotid artery. Stroke. 1976; 7:480-482.

23. Cartlidge NE, Whisnant JP, Elveback LR Carotid and vertebral-basilar transient cerebral ischemic attacks. A community study, Rochester, Minnesota. Mayo Clin Proc. 1977;52:117-120.

24. Moore DJ, Miles RD, Gooley NA, Sumner DS. Noninvasive assessment of stroke risk in asymptomatic and nonhemispheric patients with suspected carotid disease. Five-year follow-up of 294 unoperated and 81 operated patients. Ann Surg. 1985;202:491-504

25. Cantú-Brito C Ruiz-Sandoval JL, Murillo-Bonilla LM, Chiquete E, León-Jiménez C, Arauz A, et al. Acute care and one-year outcome of Mexican patients with first-ever acute ischemic stroke: the PREMIER study. Rev Neurol. 2010;51:641-649.

26. Steg PG, Bhatt DL, Wilson PW, D'Agostino R Sr, Ohman EM, Röther J, et al. One-year cardiovascular event rates in outpatients with atherothrombosis. JAMA. 2007;297:1197-1206.

27. Jeevarethinam A, Venuraju S, Dumo A, Ruano S, Rosenthal M, Nair D, et al. Usefulness of carotid plaques as predictors of obstructive coronary artery disease and cardiovascular events in asymptomatic individuals with diabetes mellitus. Am J Cardiol. 2018;121:910-916.

28. Adams A, Bojara W, Schunk K. Early diagnosis and treatment of coronary heart disease in asymptomatic subjects with advanced vascular atherosclerosis of the carotid artery (type III and IV b findings using ultrasound) and risk factors. Cardiol Res. 2018:9:22-27.

29. Mitchell CC, Korcarz CE, Tattersall MC, Gepner AD, Young RL, Post WS et al. Carotid artery ultrasound texture, cardiovascular risk factors, and subclinical arterial disease: the Multi-Ethnic Study of Atherosclerosis (MESA). Br J Radiol. 2018:91:20170637.

30. Schneider ALC, Selvin E, Sharrett AR, Griswold M, Coresh J, Jack CR, et al. Diabetes, prediabetes, and brain volumes and subclinical cerebro- 
vascular disease on MRI: the Atherosclerosis Risk in Communities Neurocognitive Study (ARIC-NCS). Diabetes Care. 2017;40:1514-1521.

31. Abraham HM, Wolfson L, Moscufo N, Guttmann CR, Kaplan RF White WB. Cardiovascular risk factors and small vessel disease of the brain: blood pressure, white matter lesions, and functional decline in older persons. J Cereb Blood Flow Metab. 2016;36:132-142.

32. Ishikawa M, Sugawara H, Tsuji T, Nagai M, Kusaka G, Naritaka H. Clinical significance of the coexistence of carotid artery plaque and white matter disease in patients with symptomatic cerebral infarction. Clin Neurol Neurosurg. 2017;163:179-185.

33. Chiquete E, Cantú-Brito C, Villarreal-Careaga J, Murillo-Bonilla LM, Rangel-Guerra R, León-Jiménez $\mathrm{C}$, et al. Obesity paradox and functional recovery in first-ever acute ischemic stroke survivors: the PREMIER study. Rev Neurol. 2010;51:705-713.

34. Chiquete E, Ruiz-Sandoval JL, Murillo-Bonilla L, León-Jiménez C, Ruiz-Madrigal B, Martínez-López E, et al. Central adiposity and mortality after first-ever acute ischemic stroke. Eur Neurol. 2013:70:117-123.

35. Streifler JY, Eliasziw M, Benavente OR, Hachinski VC, Fox AJ, Barnett HJ. Lack of relationship between leukoaraiosis and carotid artery disease. The North American Symptomatic Carotid Endarterectomy Trial. Arch Neurol. 1995;52:21-24.

36. Streifler JY, Eliasziw M, Benavente OR, Alamowitch S, Fox AJ, Hachinski V, et al. Development and progression of leukoaraiosis in patients with brain ischemia and carotid artery disease. Stroke. 2003;34:1913-1916. 